\title{
ADJUSTMENT OF THE CHARGE-DISCHARGE PARAMETERS OF A STATIONARY ENERGY STORAGE SYSTEM FOR DC ELECTRIC RAILWAYS USING PI CONTROL
}

\author{
YUKI NAKAMATA \& RYO TAKAGI \\ Kogakuin University, Japan
}

\begin{abstract}
Recent years have seen the introduction of stationary energy storage systems (SESSs) to mitigate the following problems commonly observed in DC traction power supply networks, namely: (1) the occasional loss of line receptivity for the regenerative trains when there is not enough load within the power supply network; and (2) the drop of line voltage when the traction load is too high. Commonly the charge and discharge current of an SESS is controlled using I-V characteristic controller, which determines the current depending on the terminal voltage of the SESS. However, it is known that it is a very difficult task to adjust threshold voltages such as charge start voltage. Thus, the authors propose a new approach to the determination of the voltage parameters using the PI controllers. In this research, we propose a method to adjust voltage thresholds using PI control and control method of SOE (State of Energy) to arbitrary value. Then, we propose two methods for controlling RMS current at the same time as the SOE, and we present the results of the simulation-based analyses of the proposed approach. Keywords: electric railways, traction power supply network, charge/discharge control, PI control, energy storage system, SOE (state of energy).
\end{abstract}

\section{INTRODUCTION}

Recent years have seen the increase in the number of stationary energy storage systems (SESSs) introduced in the power supply network of DC electric railways for the purpose of mitigating issues such as the large voltage fluctuation in the network or the occasional loss of receptivity of power regenerated from trains to the power supply network [1]. The effectiveness of the SESS depends heavily on the charge/discharge control strategy adopted by the SESS itself, and therefore devising a good control strategy and/or scheme for the SESS is very important. Currently, most of the SESSs in service use the I-V characteristic controllers to determine their charge/discharge currents. As shown in Fig. 1, an I-V characteristic controller determines the charge/discharge current depending on the terminal voltage of the SESS, which will fluctuate fairly widely as the distribution of train loads change [2], [3]. Generally, the threshold voltage parameters for this controller $\left(V_{a}\right.$ through $V_{f}$ in Fig. 1) are fixed during the operation of the SESS. However, it is known to be very difficult to find their appropriate values. Shiokawa and Takagi [2] attempted to optimise these parameters using the embedded simulation technique; however, the calculation time was prohibitively long, and the effects were not significant. It is clear that the values of these parameters should be variable during the operation and should be auto-adjusted according to the changes in the situation within the power supply network, e.g. the load distribution.

In this paper, the authors propose a new approach to the determination of the voltage parameters using the PI controllers and present the results of the simulation-based analyses of the proposed approach. 


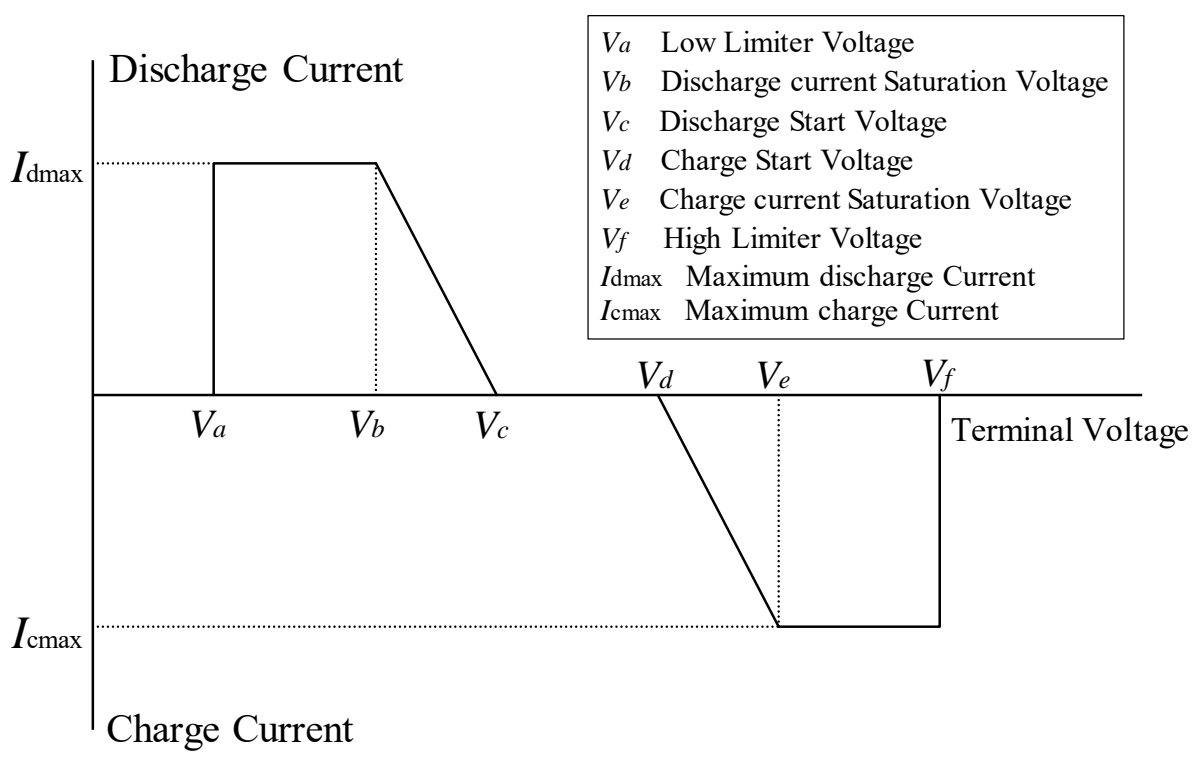

Figure 1: I-V characteristic control.

\section{ADJUSTMENT METHOD OF THE PARAMETERS USING PI CONTROL}

\subsection{The concept}

Under the I-V characteristic charge/discharge control shown in Fig. 1, raising the voltage threshold parameter values will result in less frequent charging and more frequent discharging, and therefore will lead to lower SOE (State of Energy) level of the SESS. Conversely, lowering the voltage thresholds will result in more frequent charging and less frequent discharging, and therefore will lead to higher SOE of the SESS. This fact indicates the possibility to control the SOE value by using the PI feedback controller to adjust the threshold parameter values.

Furthermore, the larger the difference between $V_{c}$ and $V_{d}$ in Fig. 1, the longer the duration during which the charge/discharge current of the SESS is zero. Also in Fig. 1, assuming that fixed values are given for $V_{c}$ and $V_{d}$, the smaller the differences between $V_{b}$ and $V_{c}$ and/or between $V_{d}$ and $V_{e}$, the larger the absolute value of the charge/discharge current of SESS at any given terminal voltage. This fact indicates the possibility to control the RMS current of the SESS by using the PI feedback controller to adjust the intervals between voltage thresholds. Controlling the current in this way is expected to yield the following effects:

- Preventing SESS overloading;

- Balancing load distribution among multiple SESSs; and

- Reducing losses in the SESS through suppression of peak charge/discharge current.

2.2 Adjusting charge/discharge control parameters by PI feedback controller

Fig. 2 shows the block diagram of the proposed controller. 


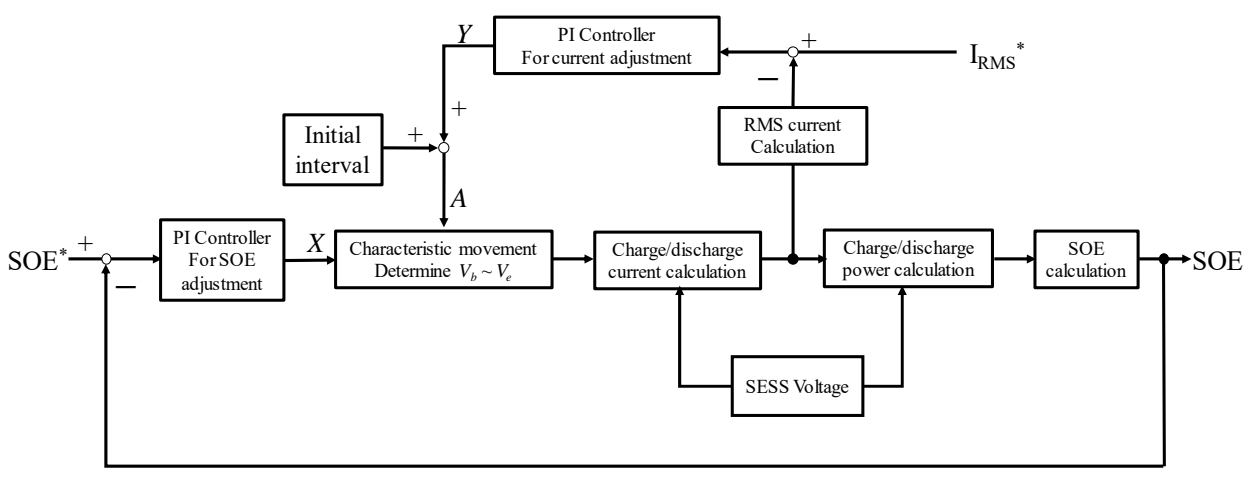

Figure 2: Block diagram of the proposed controller.

\subsubsection{Controlling the SOE}

Following the discussion in 2.1, the level of SOE of the SESS can be controlled using the strategy of moving $V_{b}$ through $V_{e}$ in Fig. 1 in parallel.

In Fig. 2, $X$ is the output of the SOE control loop. Consider the case when only this SOE control loop is activated. In this case, four of the voltage threshold parameters in Fig. $1, V_{b}$ through $V_{e}$, are to be adjusted simultaneously by using output $X$ as follows:

$$
\begin{aligned}
& V_{b}=V_{b M}-G_{X} X, \\
& V_{c}=V_{c M}-G_{X} X, \\
& V_{d}=V_{d M}-G_{X} X, \\
& V_{e}=V_{e M}-G_{X} X .
\end{aligned}
$$

As discussed in Section 2.1, by setting these voltage threshold parameters as above, they will become higher as output $X$ becomes higher causing the SOE level to fall, and they will become lower as $X$ becomes lower causing the SOE level to rise. The interval between any adjacent thresholds remain constant regardless of $X$. $V_{b M}$ through $V_{e M}$ are the medium values for $V_{b}$ through $V_{e}$ when $X=0$, and $V_{b M}<V_{c M}<V_{d M}<V_{e M}$ should hold. $G_{X}$ is the gain parameter and $G_{X}>0$.

\subsubsection{Controlling the RMS current}

Following the discussion in Section 2.1, two different control strategies can be considered for the control of the RMS current:

(i) To adjust the voltage range between $V_{c}$ and $V_{d}$ in Fig. 1, where the charging / discharging current is zero (this voltage range is hereinafter referred to as the charge/discharge inoperative region); and

(ii) To adjust the gradient of the I-V characteristics curve at the voltage range between $V_{b}$ and $V_{c}$ and between $V_{d}$ and $V_{e}$.

In Fig. 2, $A$ is the output of the current control loop, which can be used to implement either of the above strategies. The following equation is used to calculate the RMS current: 


$$
I_{R M S}(t)=\sqrt{\frac{1}{T_{W}} \int_{\tau=t-T_{W}}^{t} I_{C}^{2}(\tau) d \tau},
$$

where $T_{W}$ is the width of the temporal window for the calculation of RMS current, and $I_{C}(t)$ is the charge current at the terminal of the SESS at time $t$.

Implementation of control strategy (i) can be done as follows. First, assume that the interval between the voltage threshold parameters $V_{b}$ and $V_{c}$ is fixed to $V_{c}-V_{b}=V_{c M}-V_{b M}$ as given by eqns (1) and (2). Similarly, assume that the interval between $V_{d}$ and $V_{e}$ is fixed to $V_{e}-V_{d}=V_{e M}-V_{d M}$ as given by eqns (3) and (4). $A$ in Fig. 2 will be used to determine the interval $V_{d}-V_{c}$, which is the charge/discharge inoperative region. The larger $A$ will mean the wider charge/discharge inoperative region, and hence smaller RMS current. Using two control outputs $A$ and $X, V_{c}$ and $V_{d}$ are to be determined as follows:

$$
\begin{aligned}
& V_{c}=\frac{V_{c M}+V_{d M}}{2}-G_{X} X-\frac{A}{2}, \\
& V_{d}=\frac{V_{c M}+V_{d M}}{2}-G_{X} X+\frac{A}{2} .
\end{aligned}
$$

Implementation of control strategy ii) can be done as follows. First, assume that parameters $V_{c}$ and $V_{d}$ are to be given by (2) and (3). A in Fig. 2 will be used to determine $V_{b}$ and $V_{e}$ by assuming that $V_{c}-V_{b}=V_{e}-V_{d}=A$. The larger $A$ will mean lower gradient of the $\mathrm{V}$-I characteristics curve at these voltage ranges, which will mean smaller absolute value of the charge/discharge current and hence smaller RMS current.

\section{ASSUMPTIONS FOR THE SIMULATIONS}

The route model used for the simulation is based on an existing suburban railway. The conditions given to the simulation are as follows:

- Line: $26.577 \mathrm{~km} / 24$ stations / $1.5 \mathrm{kV}$ DC electrified;

- Substations: Five en route / no-load output voltage $1620 \mathrm{~V} /$ diode rectifiers / $6 \%$ voltage regulation;

- Trains: 8 cars/trainset / 10 trains/h;

- SESS location: Beside Substation No. 3;

- SESS rated output: 2MW (Both charge and discharge);

- $\quad$ SESS energy capacity: $388 \mathrm{kWh}$; and

- $\mathrm{I}_{\mathrm{RMS}}{ }^{*}$ in Fig. 2: $400 \mathrm{~A}$;

- Gains given to the controller: as shown in Table 1;

- Other parameters: $V_{b M}=1570 \mathrm{~V}, V_{c M}=1630 \mathrm{~V}, V_{d M}=1660 \mathrm{~V}, V_{e M}=1720 \mathrm{~V}$, $G_{X}=3$ and $T_{W}=600 \mathrm{~s}$.

Table 1: The gains given to the controller.

\begin{tabular}{|c|c|c|}
\hline PI Controller & Proportional gain & Integral gain \\
\hline For SOE adjustment & 1.17 & 0.003 \\
\hline For current adjustment & 1.00 & 0.001 \\
\hline
\end{tabular}






Figure 3: Model route map and SESS location.

\section{SIMULATION RESULTS}

\subsection{Controlling the SOE of the SESS only}

First, simulation results for cases when only the SOE control loop as explained in 2.2.1 is activated will be shown.

The following four cases of simulation have been performed.

- $\quad$ Case 1: Without PI control (no control loop is activated).

- $\quad$ Case 2: With PI control; $\mathrm{SOE}^{*}=50 \%$.

- Case 3: With PI control; $\mathrm{SOE}^{*}=50 \%$ if time $t \leq 4800 \mathrm{~s}, \mathrm{SOE}^{*}=60 \%$ otherwise.

- Case 4: With PI control; $\mathrm{SOE}^{*}=50 \%$ if time $t \leq 4800 \mathrm{~s}, \mathrm{SOE}^{*}=40 \%$ otherwise.

Fig. 4 shows the simulated SOE trajectories. Fig. 5 shows the trajectories of voltage parameters in Case 3. Fig. 6 shows the trajectories of voltage parameters in Case 4.

As shown in Fig. 4, without the PI control (Case 1) the SOE steadily decreases because there is no floating charge/discharge that pulls the SOE back to the central value (generally, the floating charge/discharge is done when the terminal voltage of the SESS is in the charge/discharge inoperative region). In contrast, in other cases (Cases 2 through 4), the PI controller successfully keeps the SOE value close to the target value of SOE*. For Cases 3 and 4 , target value will change at time $t=4800 \mathrm{~s}$, and for both cases the reaction of the SOE to the step change in $\mathrm{SOE}^{*}$ is very similar; only the direction of change is opposite. Looking at Fig. 5, the parameters temporarily change to the lower values that make the SESS "easier to charge" just after $t=4800$ s so its SOE becomes higher, but once the SOE is close to SOE* they return to the original value again so the SOE level does not change further. Similarly, looking at Fig. 6, the parameters temporarily change to the higher values that make the SESS "easier to discharge" just after $t=4800 \mathrm{~s}$ so its SOE becomes lower, but once the SOE is close to $\mathrm{SOE}^{*}$ they return to the original value again.

\subsection{Controlling both the SOE and the RMS current of the SESS at the same time}

Next, simulation results for cases when both the SOE control loop as explained in 2.2.1 and the RMS current control loop as explained in 2.2 .2 were both activated will be shown. In this 


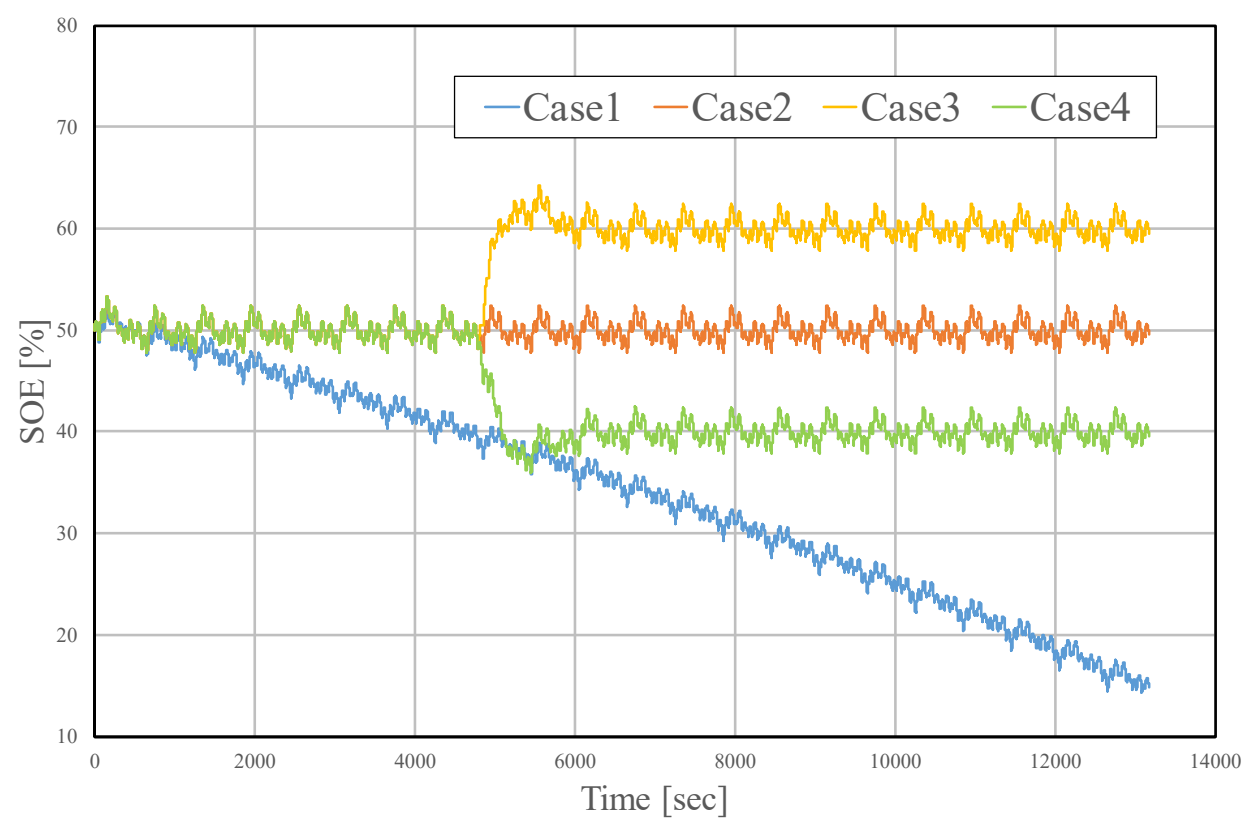

Figure 4: Simulated SOE trajectories: no PI control activated (Case 1); activating SOE control loop only (Cases 2-4).

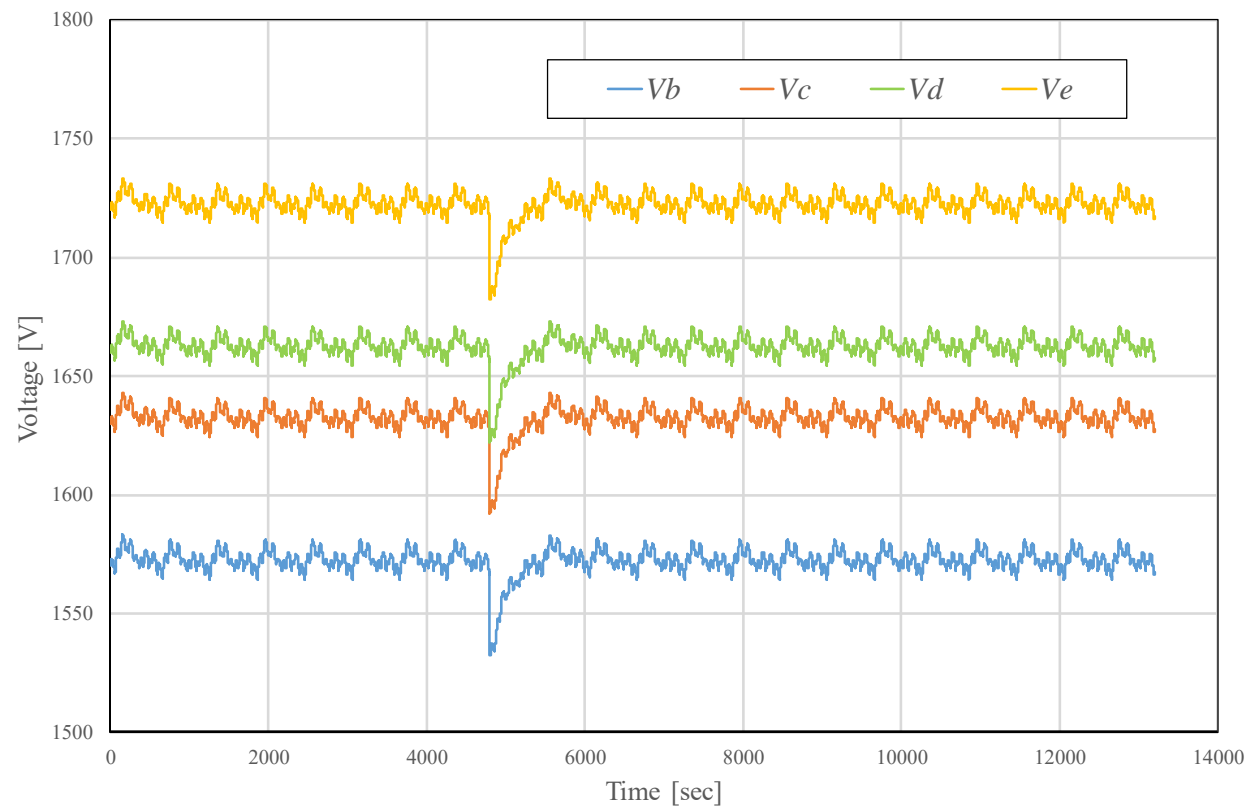

Figure 5: Simulated voltage parameter trajectories: activating SOE control loop only, Case 3. 


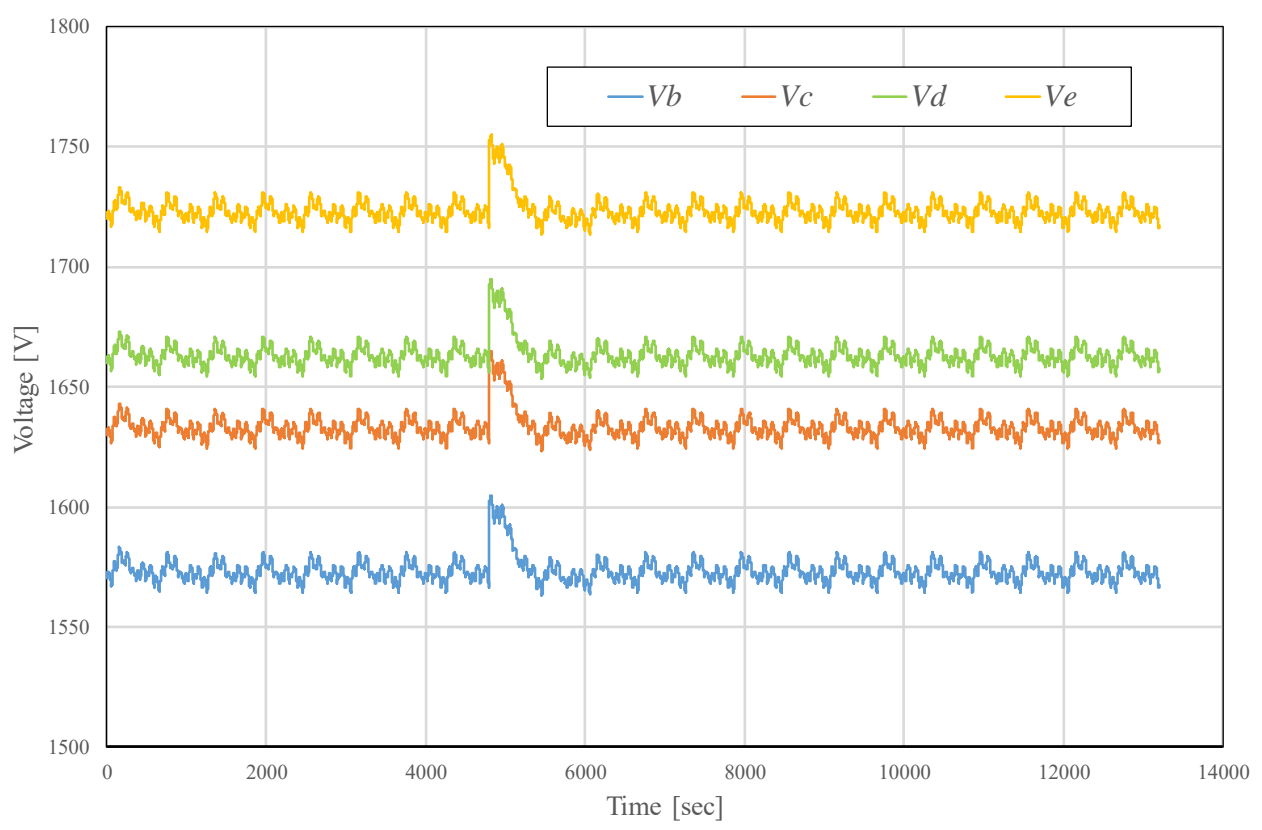

Figure 6: Simulated voltage parameter trajectories: activating SOE control loop, Case 4.

section, the SOE control loop was activated in all of the simulation attempts, and the cases where the RMS current control loop was activated and deactivated were compared. Also, for all simulation attempts it was assumed that $\mathrm{SOE}^{*}=50 \%$ if time $t \leq 4800 \mathrm{~s}$ and $\mathrm{SOE}^{*}=40 \%$ otherwise, as in Case 4 of 4.1 .

4.2.1 Strategy to change the width of the charge/discharge inoperative region The simulation results when the strategy of changing the width of the charge/discharge inoperative region was adopted will be shown in this section. Fig. 7 shows the simulated SOE trajectories. Fig. 8 shows the simulated RMS current trajectories. Fig. 9 shows the simulated voltage parameter trajectories. Note the sudden change in the RMS current observed after $t=4800 \mathrm{~s}$, caused by the assumed step change in the $\mathrm{SOE}^{*}$ from $50 \%$ to $40 \%$ there.

Fig. 7 shows that the response to the step change in $\mathrm{SOE}^{*}$ at time $t=4800 \mathrm{~s}$ becomes slightly slower when the RMS current control is added, because of the suppressed maximum charge/discharge power imposed by the RMS current control loop. As shown in Fig. 8, the RMS current is successfully suppressed by the control. However, after the convergence, the level of the RMS current became slightly lower than the target value of $\mathrm{I}_{\mathrm{RMS}}{ }^{*}=400 \mathrm{~A}$ when the RMS current control is activated. This happened because the output of the integrator would not change during the period when the RMS current was lower than the target value and therefore the width of the inoperative region remained expanded.

Initially, the width of the inoperative region $V_{d}-V_{c}=30 \mathrm{~V}$; in the end it is set to approximately $58 \mathrm{~V}$ by the controller, which is $28 \mathrm{~V}$ wider than the original, and this led to the smaller RMS current. 


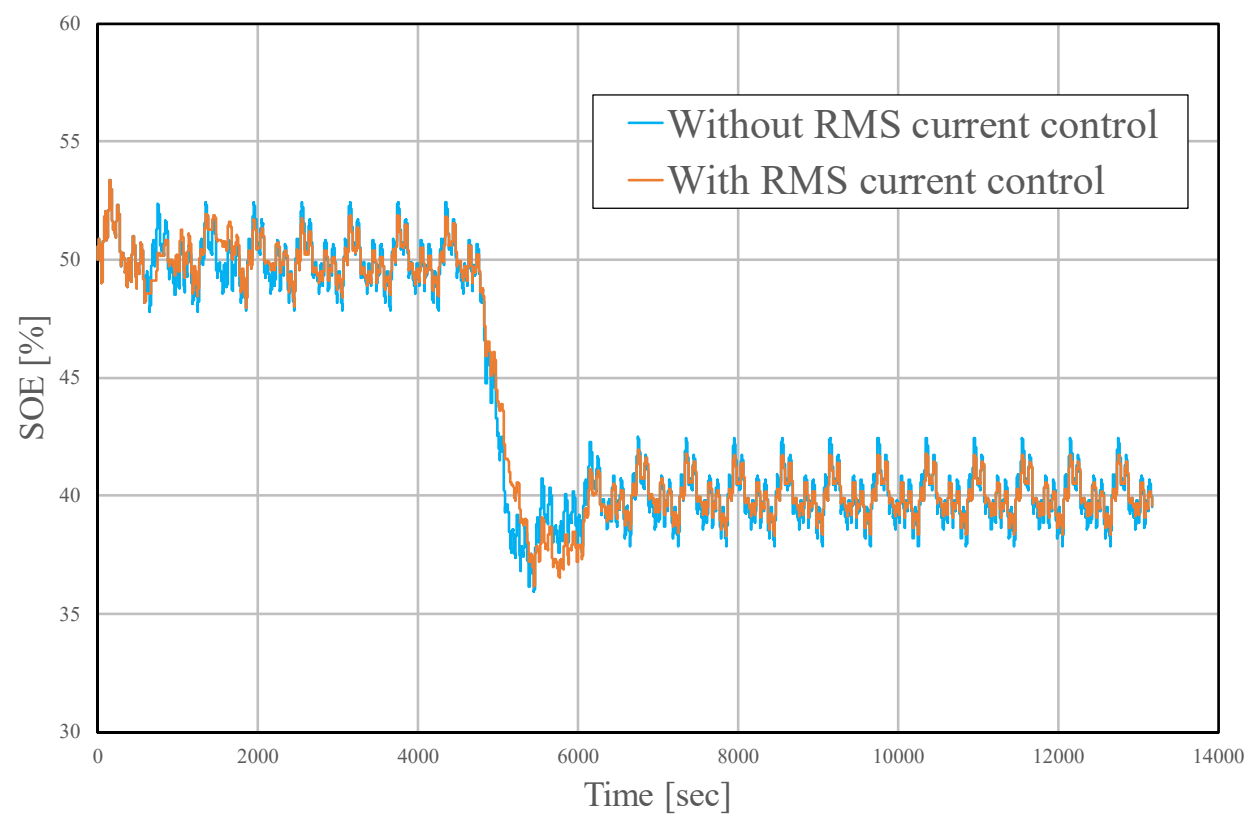

Figure 7: Simulated SOE trajectories: strategy to change the charge/discharge inoperative region width adopted for the RMS current control.

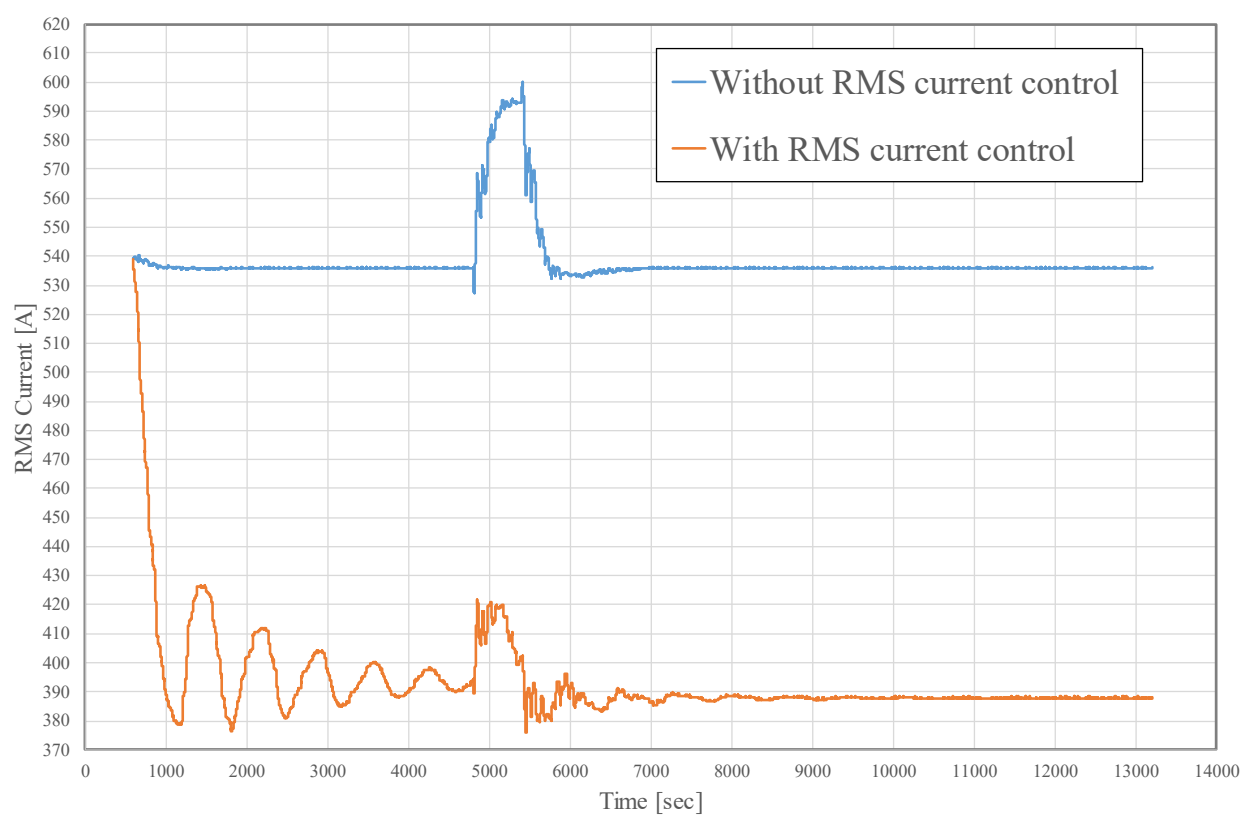

Figure 8: Simulated RMS current trajectories: strategy to change the charge/discharge inoperative region width adopted for the RMS current control. 


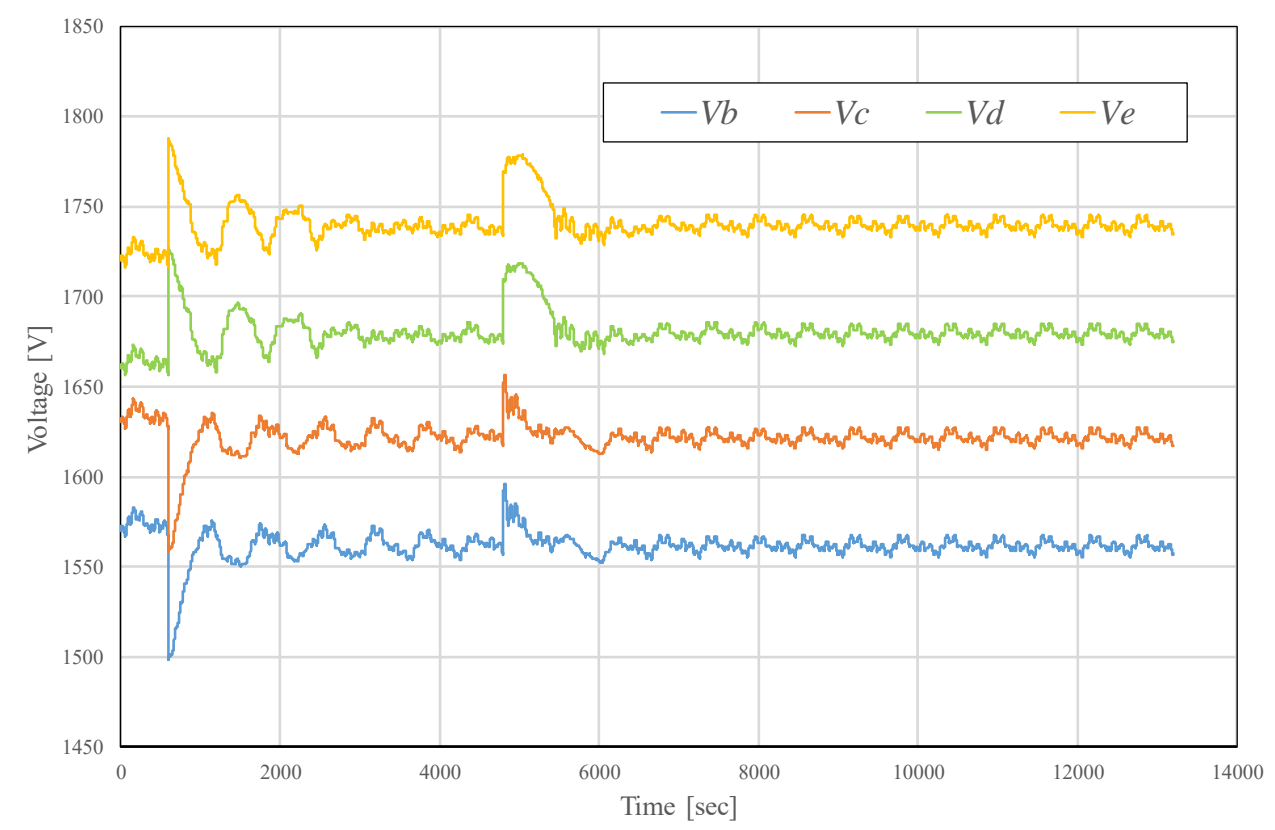

Figure 9: Simulated voltage parameter trajectories: strategy to change the charge/ discharge inoperative region width adopted for the RMS current control.

4.2.2 Strategy to change the gradient of the I-V characteristic curve

The simulation results when the strategy of changing the gradient of the I-V characteristic curve was adopted will be shown in this section. Fig. 10 shows the SOE trajectories. Fig. 11 shows the RMS current trajectories. Fig. 12 shows the voltage parameter trajectories. Note the sudden change in the RMS current observed after $t=4800 \mathrm{~s}$, caused by the assumed step change in the $\mathrm{SOE}^{*}$ from $50 \%$ to $40 \%$ there.

The results are very similar to those presented in 4.2.1. Fig. 10 shows that the response to the step change in $\mathrm{SOE}^{*}$ at time $t=4800 \mathrm{~s}$ becomes slightly slower when the RMS current control is added, because of the suppressed maximum charge/discharge power imposed by the RMS current control loop. As shown in Fig. 11, the RMS current is successfully suppressed by the control. However, after the convergence, the level of the RMS current became slightly lower than the target value of $\mathrm{I}_{\mathrm{RMS}}{ }^{*}=400 \mathrm{~A}$ when the RMS current control is activated. This happened because the output of the integrator would not change during the period when the RMS current was lower than the target value and therefore the gradient of the I-V characteristics remained gentle.

Initially, the width of the gradient regions of the I-V characteristic curve was set to $V_{c}-V_{b}=V_{e}-V_{d}=60 \mathrm{~V}$; in the end it was set to approximately $90 \mathrm{~V}$ by the controller, which is $30 \mathrm{~V}$ wider than the original, and this led to the smaller RMS current. 




Figure 10: Simulated SOE trajectories: strategy to change the gradient of the I-V characteristic curve adopted for the RMS current control.

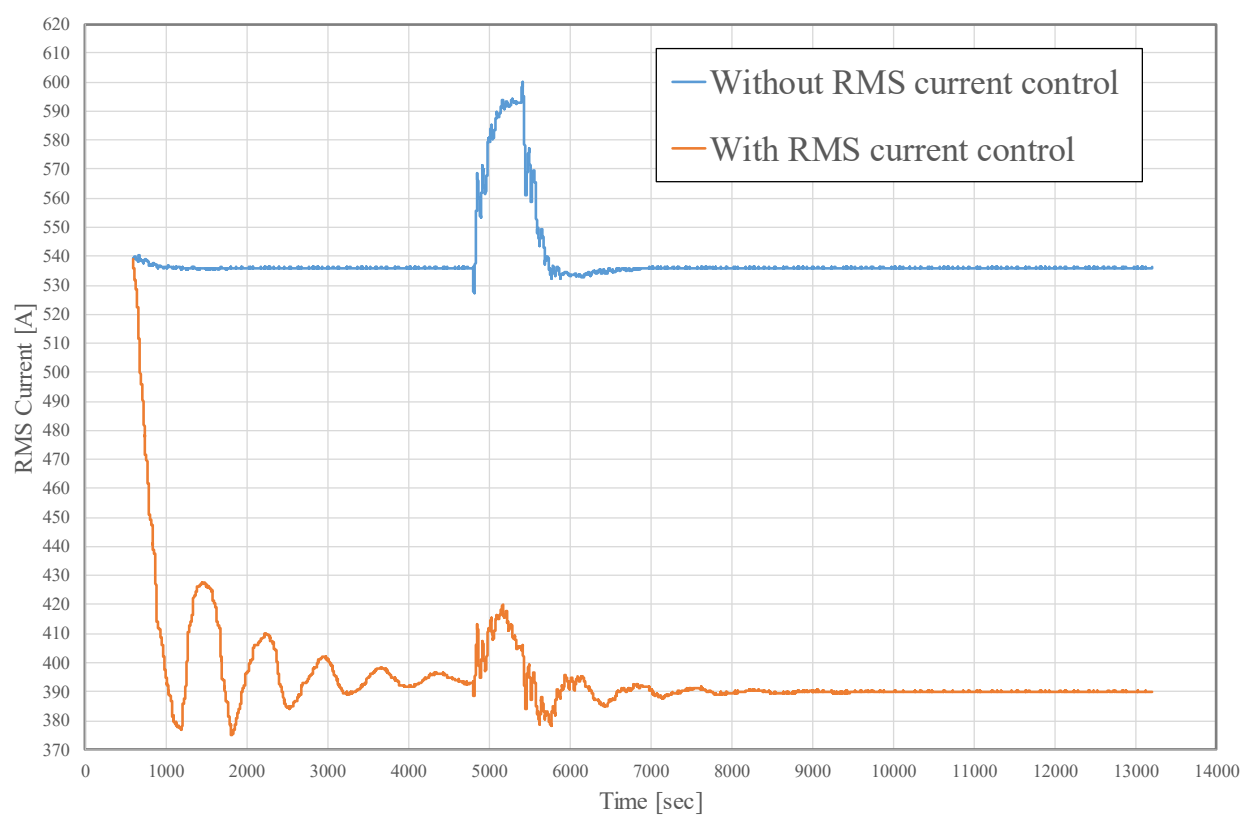

Figure 11: Simulated RMS current trajectories: strategy to change the gradient of the I-V characteristic curve adopted for the RMS current control. 


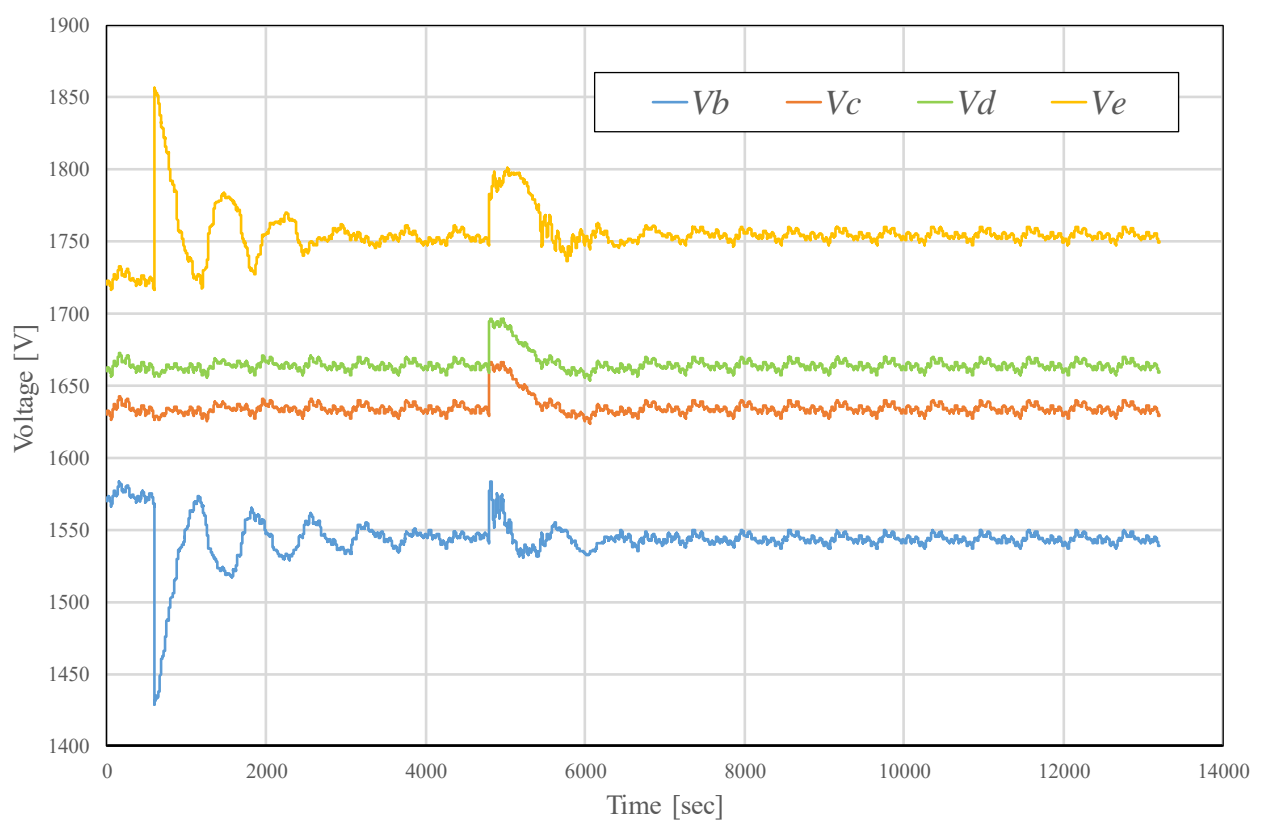

Figure 12: Simulated voltage parameter trajectories: strategy to change the gradient of the $\mathrm{I}-\mathrm{V}$ characteristic curve adopted for the RMS current control.

\section{CONCLUSIONS}

From the results of the simple simulation using the proposed control scheme, it was shown that the SOE and the RMS current of the SESS can be controlled simultaneously by automatically adjusting the voltage threshold parameters for the charge/discharge control. Two strategies for the control of RMS current produced very similar results, showing that both were very effective. However, in the control of the RMS current, the phenomenon that the RMS current converges to a value lower than the target has been observed, which should be solved by improving the controller design. In addition to this, confirmation of the control scheme by incorporating the method proposed in this research in the multi-train power network simulator is required, together with the exploration of the stability limit.

\section{REFERENCES}

[1] Sone, S., Wayside and on-board storage can capture more regenerated energy. Railway Gazette Int., 163(7), pp. 423-426, 2007.

[2] Shiokawa, K. \& Takagi, R., Numerical optimization of the charge/discharge characteristics of wayside energy storage systems by the embedded simulation technique using the railway power network simulator RTSS. Proc. COMPRAIL 2012, New Forest, UK, 2012.

[3] Konishi, T., Morimoto, H., Aihara, T. \& Tsutakawa, M., Fixed energy storage technology applied for DC electrified railway. IEEJ Trans., 5, pp. 270-277, 2010. 